\title{
Sustained Improvement in a Metastatic Colon Cancer Patient with FOLFIRI-Aflibercept after FOLFOX Failure
}

\author{
Shyam Aggarwal \\ Department of Medical Oncology, Sir Ganga Ram Hospital, New Delhi, India
}

\section{Key Words}

Colon cancer $\cdot$ FOLFIRI-aflibercept $\cdot$ FOLFOX

\begin{abstract}
The present report illustrates a case of a 37-year-old Indian male patient diagnosed with adenocarcinoma of the sigmoid colon who underwent an anterior resection with total mesorectal excision surgery. He was administered adjuvant chemotherapy with 10 cycles of a FOLFOX-4 (folinic acid, fluorouracil and oxaliplatin) regimen but developed relapse. He was then put on a FOLFIRI (folinic acid, fluorouracil and irinotecan)-aflibercept (Zaltrap) regimen and received 12 cycles during the next 6 months. During the treatment period, a reduction in ascites along with a decline in serum carcinoembryonic antigen (CEA) level was observed, though the tumor size was unchanged. After completion of 12 cycles, the patient was asymptomatic but showed signs of progression in the form of increased CEA level. The FOLFIRIaflibercept therapy was discontinued, and the patient was given supportive treatment, but he eventually died after another 6 months. The FOLFIRI-aflibercept treatment provided a progression-free survival of 6 months and an overall survival of 1 year to this patient, which corroborates the findings of the VELOUR trial.

\section{Background}

Colorectal cancer (CRC) is the third most common cancer occurring in men and the second most common among women, constituting 10 and $9.2 \%$ of total cancer cases worldwide in the respective genders. Most of the cases of CRC occur in developed regions. Around 8.5\%

KARGER 125/s $\quad \begin{aligned} & \text { Dr. Shyam Aggarwal } \\ & \text { Department of Medical Oncology } \\ & \text { Sir Ganga Ram Hospital, Rajinder Nagar } \\ & \text { New Delhi } 110060 \text { (India) } \\ & \text { E-Mail drshyam_aggarwal@yahoo.com }\end{aligned}$


Aggarwal: Sustained Improvement in a Metastatic Colon Cancer Patient with FOLFIRIAflibercept after FOLFOX Failure

of total mortality associated with cancer around the globe is ascribed to CRC [1]. In India, the prevalence of CRC is low compared to the Western world, but it is gradually increasing. Indian statistics revealed that in the year 2012, the incidence of newly diagnosed cases of CRC among men and women was 36,917 and 27,415, with mortality seen in 27,814 and 20,789 cases among the two genders, respectively [2].

It is suggested that about half of the patients diagnosed with early-stage CRC progresses to metastatic disease and about one fourth of patients present with distant metastases [3]. A 5-year survival rate among such patients remains up to $10 \%$, in spite of several chemotherapeutic regimens available. In recent years, the treatment approach for metastatic CRC (mCRC) has been realigned towards the use of biological targeted therapies along with the use of cytotoxic regimens. Among such approaches, the use of antiangiogenic agents that specifically target and block the proangiogenic factor vascular endothelial growth factor (VEGF)-A pathway has shown improvement in antitumor response and survival rates. The humanized monoclonal antibody bevacizumab was the first antiangiogenic agent that was approved to be used in cancer treatment and was found to be successful in mCRC treatment by inhibiting the VEGF signaling and angiogenesis [4].

In 2012, the United States Food and Drug Administration (FDA) approved another antiangiogenic agent named aflibercept for the treatment of mCRC. Aflibercept, a novel antiangiogenic agent, is a fully human, soluble decoy receptor protein for VEGF-A. It has been engineered by fusing the immunoglobulin (Ig) domain of human VEGFR-1 and VEGFR-2 with the constant region of human IgGI. The drug acts as decoy receptor and binds with circulating VEGF and prevents its association with physiological VEGFR-1 and VEGFR-2, thus completely blocking the VEGF-A-induced cell proliferation and angiogenesis [5]. In preclinical binding kinetics studies, aflibercept has been reported to have approximately 100 -fold binding affinity towards VEGF-A compared to bevacizumab or ranibizumab. Additionally, placental growth factor was also found to bind with high affinity with aflibercept, but not at all with either bevacizumab or ranibizumab. Aflibercept also showed markedly more potent blockade of VEGFR-1 or VEGFR-2 activation than either of the above anti-VEGF-A antibodies [6].

VELOUR, a randomized double-blind phase III trial, was a landmark study about the use of aflibercept in mCRC patients who presented either resistance or progression to prior oxaliplatin-containing regimens. Enrolled patients received a combination of either a FOLFIRIaflibercept or FOLFIRI-placebo regimen. The results of the study revealed that the overall survival (OS) was statistically greater (median of 13.5 months) in the FOLFIRI-aflibercept arm compared to 12.1 months seen in the FOLFIRI-placebo arm (HR: 0.817, 95\% CI: $0.713-$ $0.937 ; p=0.0032$ ). A significant improvement was also observed in progression-free survival (PFS; median 6.9 vs. 4.7 months; HR: 0.758, 95\% CI: 0.661-0.869; $\mathrm{p}<0001$ ) with FOLFIRIaflibercept compared to the FOLFIRI-placebo treatment. Following the VELOUR trial, aflibercept in combination with FOLFIRI emerged as the first agent that showed survival benefits among mCRC patients who were resistant to or have progressed following an oxaliplatincontaining regimen [7]. This study further led to FDA and European Medicines Agency approval of aflibercept use in combination with other chemotherapeutic agents namely FOLFIRI (folinic acid, 5-flurouracil and irinotecan) to treat such specific populations of mCRC patients [8].

The present report describes the use of a FOLFIRI-aflibercept combination regimen in a patient of mCRC who relapsed after a FOLFOX-4 regimen of 10 cycles after laparoscopic anterior resection with total mesorectal excision surgery. This is the first ever reported case study on the use of aflibercept in an Indian patient. The observations of this case would provide an insight into the safety and efficacy of aflibercept among Indian mCRC patients with peritoneal involvement and ascites, who are often resistant to chemotherapy. 
Aggarwal: Sustained Improvement in a Metastatic Colon Cancer Patient with FOLFIRIAflibercept after FOLFOX Failure

\section{Case Report}

History

A 37-year-old male was diagnosed with adenocarcinoma of the sigmoid colon with positive lymph nodes in July 2012 following a colonoscopy. A primary histopathology report suggested poorly differentiated adenocarcinomas in the sigmoid colon with infiltration of the lamina propria by sheets of signet ring cells with a marked degree of pleomorphism and anaplasia. Further, contrast-enhanced computed tomography (CECT) of the whole abdomen also revealed fatty infiltration of the liver with circumferential wall thickening in the sigmoid colon and rectosigmoid junction with extensive pericolonic fat stranding. A biochemical estimation of serum carcinoembryonic antigen (CEA) level, done in June 2012, was found to be $28 \mathrm{ng} / \mathrm{ml}$.

Following this diagnosis, a laparoscopic anterior resection with total mesorectal excision surgery was performed in July 2012. The histopathology report confirmed the presence of mucin-secreting adenocarcinomas in the colon with metastasis to 16 lymph nodes with pT3pN2 staging. The postoperative CEA level was found to be $19.55 \mathrm{ng} / \mathrm{ml}$. The patient was then administered adjuvant chemotherapy with 10 cycles of a FOLFOX-4 regimen starting from August 2012 and lasting until December 2012. The patient tolerated the chemotherapy well. During chemotherapy, the serum CEA levels declined to $9.4 \mathrm{ng} / \mathrm{ml}$ (November 2012). In January 2013, the patient reported difficulty in breathing, ascites and intermittent pain in the abdomen for 1 week.

\section{Investigations}

A postoperative CECT of the whole abdomen was conducted in January 2013 and showed ascites with omental infiltrates suggestive of disseminated malignancy and minimal right pleural effusion with infiltrates seen in the right lung base. Paracentesis was performed in January 2013 and showed malignant positive cells. Simultaneous serum CEA determination showed levels of $278 \mathrm{ng} / \mathrm{ml}$. The postoperative positron emission tomography (PET) scan done at the same time revealed metabolically inactive fluid collection in the perihepatic, subhepatic, hepatorenal perisplenic and bilateral paracolic gutters and the pelvic cavity, metabolically inactive diffuse irregular omental thickening with fat stranding, and mild right pleural effusion with low-grade fludeoxyglucose (FDG) uptake in the right lower lobe.

Treatment

Considering these observations, it was concluded that the patient had developed relapse of colon carcinoma, and he was advised a FOLFIRI regimen with aflibercept for further management. The patient was provided with aflibercept (Zaltrap) by Sanofi through the Named Patient Program in India. The patient was prescribed 12 cycles of a FOLFIRI-aflibercept regimen starting from January 2013, with each cycle (leucovorin $400 \mathrm{mg} / \mathrm{m}^{2}$ and irinotecan 180 $\mathrm{mg} / \mathrm{m}^{2}$ on day 1,5 -fluorouracil $2,400 \mathrm{mg} / \mathrm{m}^{2}$ over $48 \mathrm{~h}$ and Zaltrap $4 \mathrm{mg} / \mathrm{kg}$ q2w on day 1 ) administered at an interval of 2 weeks. After 3 months (April 2013) of initiating the FOLFIRIaflibercept regimen, the serum CEA level fell to $36.01 \mathrm{ng} / \mathrm{ml}$, and treatment was continued.

After 6 months of the FOLFIRI-aflibercept therapy, a PET scan was performed in July 2013, which showed low-grade FDG uptake in multiple enlarged hypodense discrete and conglomerate lymph nodes in infrarenal, para-aortic and aortocaval groups, low-grade FDG uptake in diffuse irregular thickening and fat stranding of the omentum, metabolically inactive small fluid attenuation cyst in the right lobe segment VII, and mild fluid collection in the right anterior and subhepatic space. In comparison to the previous PET scan, it was found that there was resolution of the right pleural effusion and collapse as well as consolidation 
Aggarwal: Sustained Improvement in a Metastatic Colon Cancer Patient with FOLFIRIAflibercept after FOLFOX Failure

with partial resolution of ascites, but no new lesion was observed. However, at this juncture, the serum CEA level started rising and attained a level of $171.69 \mathrm{ng} / \mathrm{ml}$. The treatment was discontinued after this as the patient showed signs of progressive disease despite being asymptomatic. A CEA test done in the month of September 2013 revealed levels up to 616.21 $\mathrm{ng} / \mathrm{ml}$, which further rose to $1,092.35 \mathrm{ng} / \mathrm{ml}$ in a diagnostic test performed in October 2013. A concurrent whole-body PET scan revealed extensive omental, serosal and mesenteric deposits with multiple mildly FDG-avid pelvic, retroperitoneal lymph nodes with extensive skeletal metastases and mild ascites. In comparison to the previous PET scan and serum CEA levels, these observations were suggestive of disease progression with mild improvement in ascites. The patient eventually died in January 2014.

The patient was compliant in taking the treatment; however, a delay of 6-7 days was observed in administering the chemotherapy due to grade II neutropenia observed in the patient during the last 3 treatment cycles. During the entire treatment period with FOLFIRIaflibercept, the patient did not experience any treatment-related adverse events, namely hypertension, diarrhea and proteinuria, or did not have any other comorbid conditions.

\section{Discussion}

Angiogenesis plays a crucial role in tumor growth and progression of CRC. These tumors thrive on the oxygen and nutrients provided by the circulating blood; thus, the vasculature within the tumors and its vicinity is critical for its development and growth [9]. Several growth factors including VEGF have been ascribed to neovascularization among such tumors. Studies have demonstrated that VEGF expression was found to be elevated among different types of tumors including CRC. This elevated expression of VEGF has been further associated with tumor progression, invasion and metastasis of CRC [10].

Targeting angiogenesis via inhibition of growth factors like VEGF and epidermal growth factor seems to be a plausible treatment for CRC. In recent years, several treatment options have been developed to arrest the angiogenic process by targeting biological molecules to treat CRC, which includes anti-VEGF drugs like bevacizumab and aflibercept and most recently, the multi-kinase inhibitor regorafenib. Among these agents, bevacizumab was the first drug to be approved and used for mCRC as first- and second-line treatment. However, lately, aflibercept therapy has been approved by the FDA in mCRC patients after failure of oxaliplatin-based treatment. It has a better binding capacity with VEGF (both VEGF-A and -B) and when combined with a FOLFIRI regimen has been found to be effective by improving OS and PFS in patients showing resistance and/or progression to prior an oxaliplatin-based regimen independently of prior treatment with bevacizumab or not.

The patient in the present case was a suitable candidate for a FOLFIRI-aflibercept regimen as he had shown progression of disease after 10 cycles of the FOLFOX-4 regimen, and this profile was similar to about $10 \%$ of the population enrolled in the VELOUR study [7]. It was observed that the FOLFIRI-aflibercept treatment kept the patient asymptomatic and progression free for almost 6 months and further exhibited partial resolution of cancerassociated ascites. Ascites is one of the cardinal features of metastatic cancers and a grave prognostic sign which can add to the patient's distress. VEFG plays an important role in ascites formation by promoting vascular permeability $[11,12]$. By blocking the actions of VEGF, aflibercept has also been reported to reduce the ascites formation in addition to controlling tumor growth [13]. Previous studies on ovarian cancer also documented that aflibercept significantly controlled the ascites formation $[14,15]$. In our case report, we did not perform paracenteses, but PET scan results corroborate the findings of the studies mentioned above. 
Aggarwal: Sustained Improvement in a Metastatic Colon Cancer Patient with FOLFIRIAflibercept after FOLFOX Failure

The patient reported symptomatic improvement during this period; however, the cancer showed progression after 6 months, and the patient eventually died 12 months after therapy initiation. In this case, the PFS and the OS period were about 6 months and 1 year, respectively, with FOLFIRI-aflibercept treatment. These findings were in line with the observations of the VELOUR study, where the median PFS was 6.9 months and the median OS was 13.5 months among the participants receiving FOLFIRI-aflibercept therapy [7].

Overall, this case study is the first available report on the use of aflibercept in an Indian patient with mCRC. Aflibercept treatment was found to prolong the PFS by up to 6 months in the patient after failure of the FOLFOX-4 regimen, without developing any adverse events and being well tolerated by the patient. Though the patient eventually progressed and died, the survival time was extended by up to 1 year. The results of this case study suggest that aflibercept along with FOLFIRI can improve the outcome/survival (both PFS and OS) of patients with metastatic colorectal carcinoma who have already progressed from an oxaliplatin-based regimen. In this case, we did not notice any reduction in tumor size during the treatment, despite observing a decline in CEA levels until 6 months from therapy initiation. In the VELOUR study also, stable disease was reported in almost $66 \%$ of patients treated with FOLFIRI-aflibercept combination. Additionally, the patient did not experience any serious adverse events that are usually associated with aflibercept therapy. Collectively, FOLFIRI-aflibercept chemotherapy seems to be a promising regimen for Indian patients of mCRC, with a good efficacy and safety profile. However, more clinical reports from routine practice will help in developing the actual efficacy and safety profile of this combination.

\section{Acknowledgement}

I would like to acknowledge Sanofi India for their editorial support in preparing the manuscript. Also, I would like to thank Dr. Ashok Krishna, Medical Affairs, Sanofi, for coordinating with me on behalf of Sanofi India.

\section{Statement of Ethics}

I hereby declare that written informed consent was obtained from the patient's father for publication of this case report and accompanying images since the patient has died.

\section{Disclosure Statement}

No potential conflicts of interest in this study were reported.

\section{References} Accessed February 24, 2014.

3 Bartlett DL, Chu E: Can metastatic colorectal cancer be cured? Oncology (Williston Park) 2012;26:266-275.

4 McRee AJ, Goldberg RM: Optimal management of metastatic colorectal cancer: current status. Drugs 2011;71:869-884.

5 Patel A, Sun W: Ziv-aflibercept in metastatic colorectal cancer. Biologics 2014;8:13-25. 


\section{Case Reports in Oncology}

\begin{tabular}{l|l}
\hline Case Rep Oncol 2015;8:487-492 \\
\hline DOI: 10.1159/000441413 & $\begin{array}{l}\text { (c) 2015 The Author(s). Published by S. Karger AG, Basel } \\
\text { www.karger.com/cro }\end{array}$ \\
\hline
\end{tabular}

Aggarwal: Sustained Improvement in a Metastatic Colon Cancer Patient with FOLFIRIAflibercept after FOLFOX Failure

6 Papadopoulos N, Martin J, Ruan Q, Rafique A, Rosconi MP, Shi E, et al: Binding and neutralization of vascular endothelial growth factor (VEGF) and related ligands by VEGF Trap, ranibizumab and bevacizumab. Angiogenesis 2012;15:171-185.

-7 Van Cutsem E, Tabernero J, Lakomy R, Prenen H, Prausova J, Macarulla T, et al: Addition of aflibercept to fluorouracil, leucovorin, and irinotecan improves survival in a phase III randomized trial in patients with metastatic colorectal cancer previously treated with an oxaliplatin-based regimen. J Clin Oncol 2012;30:3499-3506.

8 Aflibercept approval by FDA for mCRC. Available at http://www.fda.gov/Drugs/InformationOnDrugs/ApprovedDrugs/ucm314438.htm. Accessed January 14, 2014.

-9 Zetter BR: Angiogenesis and tumor metastasis. Annu Rev Med 1998;49:407-424.

$\rightarrow 10$ Ishigami SI, Arii S, Furutani M, Niwano M, Harada T, Mizumoto M, et al: Predictive value of vascular endothelial growth factor (VEGF) in metastasis and prognosis of human colorectal cancer. Br J Cancer 1998;78:1379-1384.

11 Zebrowski BK, Liu W, Ramirez K, Akagi Y, Mills GB, Ellis LM: Markedly elevated levels of vascular endothelial growth factor in malignant ascites. Ann Surg Oncol 1999;6:373-378.

$\checkmark 12$ Kraft A, Weindel K, Ochs A, Marth C, Zmija J, Schumacher P, et al: Vascular endothelial growth factor in the sera and effusions of patients with malignant and nonmalignant disease. Cancer 1999;85:178-187.

-13 Byrne AT, Ross L, Holash J, Nakanishi M, Hu L, Hofmann JI, et al: Vascular endothelial growth factor-trap decreases tumor burden, inhibits ascites, and causes dramatic vascular remodeling in an ovarian cancer model. Clin Cancer Res 2003;9:5721-5728.

14 Colombo N, Mangili G, Mammoliti S, Kalling M, Tholander B, Sternas L, et al: A phase II study of aflibercept in patients with advanced epithelial ovarian cancer and symptomatic malignant ascites. Gynecol Oncol 2012;125:42-47.

15 Gotlieb WH, Amant F, Advani S, Goswami C, Hirte H, Provencher D, et al: Intravenous aflibercept for treatment of recurrent symptomatic malignant ascites in patients with advanced ovarian cancer: a phase 2 , randomised, double-blind, placebo-controlled study. Lancet Oncol 2012;13:154-162. 